\title{
Spectral Methods for Time-Dependent Studies of Accretion Flows. I. Two-dimensional, Viscous, Hydrodynamic Disks
}

\author{
Chi-kwan Chan, Dimitrios Psaltis, and Feryal Özel ${ }^{1}$ \\ Physics and Astronomy Departments, University of Arizona, 1118 E. 4th St., Tucson, AZ 85721
}

\begin{abstract}
We present a numerical method for studying the normal modes of accretion flows around black holes. In this first paper, we focus on two-dimensional, viscous, hydrodynamic disks in pseudo-Newtonian potentials, for which the linear modes have been calculated analytically in previous investigations. We use a pseudo-spectral algorithm to evolve the hydrodynamic equations and devise a number of test problems to validate its implementation. We demonstrate the ability of the spectral method to handle accurately non-reflective boundary conditions and to reproduce correctly the stability criteria for differentially rotating hydrodynamic flows.
\end{abstract}

Subject headings: accretion disks, black hole physics, hydrodynamics

\section{Introduction}

Observations of accretion flows around black holes in galactic systems and in the centers of galaxies show strong evidence for the presence of long-lived, global modes at characteristic frequencies of the black-hole spacetimes (see van der Klis 2000; McClintock and Remillard 2004, for reviews). The origin of these modes is still a matter of debate (see, e.g., Psaltis 2001, 2004). It is anticipated, however, that modeling accurately their origin and physical properties will provide the first measurements of the spins of black holes in the universe as well as direct tests of strong-field general relativity (Psaltis 2004).

Systematic studies of modes in accretion disks around black holes have so far been mostly analytical, performed in the linear regime for flows with an artificial viscosity law (see Wagoner 1999; Kato 2001 and reference therein; see, however, Gammie \& Balbus 1994 and Menou 2003 for studies of MHD disks). These studies have shown the presence of three types of trapped global modes, namely inertial-gravity, corrugation, and inertial-pressure modes, with properties that appear to agree with observations (see Wagoner et al. 2001).

Despite their success, analytical models have several limitations. First, they rely on a number of approximations, such as the WKB approximation in the radial direction. Moreover, because

\footnotetext{
${ }^{1}$ Hubble Fellow
} 
only linear terms in perturbations are taken into account, these studies are unable to reproduce resonances and couplings between various modes. However, the detected QPOs often have fractional amplitudes as large as $40 \%$ of the total X-ray flux, making the linear approximation inaccurate (see, e.g., McClintock and Remillard 2004). Furthermore, pairs of QPOs are typically observed (see Strohmayer 2001a,b) with frequency ratios equal to ratios of small integers, strongly arguing for the presence of resonances between modes (Abramowicz, \& Kluźniak 2001). Finally, analytical and numerical studies of oscillations in MHD disks cast doubt on the presence of oscillations at the radial epicyclic frequencies, as required by all diskoseismic calculations (see, e.g., Hawley, \& Krolik 2001, 2002; Armitage and Reynolds 2003). Answering all the above questions necessitates a systematic, numerical study of accretion disk oscillations.

Numerical studies of accretion disk modes have been very few and have not addressed in detail the above issues (see, e.g., Chen, \& Taam 1994, 1995; Milsom, \& Taam 1996, 1997, for simulations of viscous disks). In fact, no numerical simulation of hydrodynamic disks to date has resulted in solutions that show all three types of accretion-disk modes that were discovered analytically. In this series of papers, we perform a systematic study of the oscillatory behavior of hydrodynamic and MHD accretion disks in two and three dimensions. To this end, we have developed a pseudospectral numerical algorithm, which we present here. Even though spectral methods have been used extensively in the study of hydrodynamic modes in various planetary atmospheres, including the earth's (see, e.g., Mote, \& O'Neill 2000), their application to the studies of accretion disks has been very limited (Godon 1997).

Spectral algorithms are high order numerical methods and are perfectly suited to studying modes of hydrodynamic flows for a number of reasons. Their major advantage is accuracy and economy in the number of degrees of freedom (Bonazzola et al. 1998). In spectral methods, only $\sim \pi$ collocation points are necessary to resolve one wavelength (Boyd 2000), whereas in finite difference methods a lot more grid points are required to reach the same accuracy. The solution is already expressed as a set of orthonormal modes, which, at least in the azimuthal direction, are also the eigenmodes of the system; this property improves the accuracy of studies of mode coupling and resonance. Moreover, the dimensionality of the problem can be altered very easily, for investigating the effects of the number of spatial dimensions on the properties of hydrodynamic modes and turbulence. Finally, in simulating MHD flows, the high order of spectral methods allows for the equations to be solved in terms of the vector potential and not of the magnetic field itself; this guarantees that the resulting magnetic field is always divergence free.

Naturally, when discontinuities are present, simple spectral methods fail to reproduce piecewise continuous solutions and oscillations appear around the discontinuities (Gibbs phenomenon). Even though this is not an issue in studying viscous accretion disks with an artificially increased value of the viscosity, it is an important consideration when modeling MHDs disk without viscosity. However, it has been shown that spectral methods may be designed to give correct shock characteristics for piecewise discontinuous problems (Don 1994; Don and Quillen 1995; Guo et al. 2001; Li 2001). We will address this issue in the second paper of this series. 
In this first paper, we discuss our numerical algorithm for evolving two-dimensional viscous, hydrodynamic accretion disks. In the following section, we present our assumptions and equations and, in $\S 3$, we discuss the details of our numerical method. Finally, we have performed a series of tests to validate our algorithm, which we present in $\S 4$.

\section{Assumptions}

We consider two-dimensional hydrodynamics with pressure and shear viscosity. We neglect the self-gravity, bulk viscosity, and magnetic field of the flow. The hydrodynamic equations, therefore, contain the continuity equation

$$
\frac{\partial \Sigma}{\partial t}+\nabla \cdot(\Sigma \mathbf{v})=0
$$

and the two-dimensional Navier-Stokes equation

$$
\frac{\partial \mathbf{v}}{\partial t}+(\mathbf{v} \cdot \nabla) \mathbf{v}=-\frac{1}{\Sigma} \nabla P-\nabla \Phi+\frac{1}{\Sigma} \nabla\left(\nu_{s} \Sigma \sigma\right),
$$

where $\Sigma$ is the height-integrated density and $\mathbf{v}$ is the velocity of the flow. The height-integrated pressure $P$ is given by the equation of state $P=K \Sigma^{\Gamma}$ and we denote the sound speed by $c_{s} \equiv \sqrt{\Gamma K \Sigma^{\Gamma-1}}$. We use the pseudo-Newtonian potential $\Phi(r)=G M /\left(r-r_{g}\right)$, where $G$ is the gravitational constant, $M$ is the central object mass, and $r_{g}$ is the Schwarzschild radius of the central object. We denote by $\nu_{s}$ the height-averaged shear viscosity coefficient and by $\sigma$ the viscosity stress tensor.

Writing the equations in polar coordinates $(r, \phi)$, we have

$$
\begin{aligned}
\frac{\partial \Sigma}{\partial t}= & -\left[\frac{1}{r} \frac{\partial}{\partial r}\left(r \Sigma v_{r}\right)+\frac{1}{r} \frac{\partial}{\partial \phi}\left(\Sigma v_{\phi}\right)\right], \\
\frac{\partial v_{r}}{\partial t}=- & {\left[v_{r} \frac{\partial v_{r}}{\partial r}+\frac{v_{\phi}}{r} \frac{\partial v_{r}}{\partial \phi}-\frac{v_{\phi}^{2}}{r}\right]-\frac{d \Phi}{d r}-\left[\frac{c_{s}^{2}}{\Sigma} \frac{\partial \Sigma}{\partial r}\right] } \\
+ & {\left[\frac{1}{\Sigma r} \frac{\partial}{\partial r}\left(\nu_{s} r \Sigma \sigma_{r r}\right)+\frac{1}{\Sigma r} \frac{\partial}{\partial \phi}\left(\nu_{s} \Sigma \sigma_{r \phi}\right)-\frac{\sigma_{\nu_{s} \phi \phi}}{r}\right], } \\
\frac{\partial v_{\phi}}{\partial t}=- & {\left[v_{r} \frac{\partial v_{\phi}}{\partial r}+\frac{v_{\phi}}{r} \frac{\partial v_{\phi}}{\partial \phi}+\frac{v_{r} v_{\phi}}{r}\right]-\left[\frac{c_{s}^{2}}{\Sigma r} \frac{\partial \Sigma}{\partial \phi}\right] } \\
+ & {\left[\frac{1}{\Sigma r} \frac{\partial}{\partial r}\left(r \nu_{s} \Sigma \sigma_{r \phi}\right)+\frac{1}{\Sigma r} \frac{\partial}{\partial \phi}\left(\nu_{s} \Sigma \sigma_{\phi \phi}\right)+\frac{\nu_{s} \sigma_{r \phi}}{r}\right], }
\end{aligned}
$$

where the different components of the viscosity tensor are

$$
\begin{aligned}
\sigma_{r r} & =\frac{4}{3} \frac{\partial v_{r}}{\partial r}-\frac{2}{3} \frac{v_{r}}{r}-\frac{2}{3 r} \frac{\partial v_{\phi}}{\partial \phi}, \\
\sigma_{r \phi} & =\sigma_{\phi r}=\frac{\partial v_{\phi}}{\partial r}-\frac{v_{\phi}}{r}+\frac{1}{r} \frac{\partial v_{r}}{\partial \phi}, \\
\sigma_{\phi \phi} & =-\frac{2}{3} \frac{\partial v_{r}}{\partial r}+\frac{4}{3} \frac{v_{r}}{r}+\frac{4}{3 r} \frac{\partial v_{\phi}}{\partial \phi} .
\end{aligned}
$$


When we consider problems related to the inner parts of accretion disks, we scale all the variables with quantities that depend linearly on the central object mass $M$. Numerically, this is equivalent to setting $G M=1$. The length scale in these problems is comparable to the Schwarzschild radius so the natural choice of unit for length is $G M / c^{2}$; the corresponding time scale is $G M / c^{3}$ and the velocity scale is $c$. The strength of the pressure term is determined by $c_{s}^{2}$, which simply has units of $c^{2}$, whereas the viscosity coefficient $\nu_{s}$ is in units of $G M / c$. For geometrically thin disks, the typical dimensionless pressure and viscosity have numerical values around $10^{-4}$. The scale of the column density $\Sigma$ does not enter our equations.

When we study the viscous spreading of a ring, the length scale is much larger than the Schwarzschild radius and we recover Newtonian gravity. In this kind of problem, the length scale is the initial radius of the ring $R_{0}$. The natural choice of time scale and velocity scale are $1 / \Omega_{K}\left(R_{0}\right)=$ $\sqrt{R_{0}^{3} / G M}$ and $v_{\phi}\left(R_{0}\right)=\sqrt{G M / R_{0}}$. The pressure term will therefore scale with $G M / R_{0}$ and the viscosity will scale with $\sqrt{G M R_{0}}$. Again, the scale of $\Sigma$ does not enter the equations.

\section{Numerical Method}

In our numerical algorithm, we use pseudo-spectral methods to evaluate the spatial derivatives in the partial differential equations and an explicit Runge-Kutta method to advance the solution in time.

Spectral methods are based on the idea that any function can be expanded as a series of orthonormal functions. When well-behaved functions are chosen as the basis, these series converge exponentially in the absence of discontinuities and the numerical partial derivatives can be trivially evaluated. There exist different ways to approximate functions by a series with a finite number of terms, such as the Galerkin method, the tau method, and the pseudo-spectral method (see Canuto 1988; Gottlieb and Orszag 1983; Guo 1998; Boyd 2000; Peyret 2002). In pseudo-spectral methods, as the one we employ here, we choose a set of collocation points and then evaluate the expansion (spectral) coefficients such that the truncated series agree exactly with the original functions at the collocation points. In the next subsection, we discuss our implementation of the pseudo-spectral method.

\subsection{Collocation Methods}

The Fourier basis is the natural choice for expanding functions with periodic boundary conditions, as is the case along the azimuthal direction in our problem. Any physical quantity $f=f(r, \phi)$ can be expanded in this basis as

$$
f(r, \phi)=\sum_{m=-\infty}^{\infty} \hat{f}_{m}(r) e^{i m \phi} .
$$


We use the discrete collocation points

$$
\phi_{j}=\frac{2 \pi j}{M}, \quad \text { for }-\frac{M}{2} \leq j \leq \frac{M}{2}-1
$$

so that the above Fourier series can be approximated by the (truncated) discrete Fourier series

$$
f\left(r, \phi_{j}\right)=\sum_{m=-M / 2}^{M / 2-1} \hat{f}_{m}(r) e^{i 2 \pi m j / M} .
$$

The derivative of $f$ with respect to $\phi$ is, therefore,

$$
\left.\frac{\partial f}{\partial \phi}\right|_{\phi_{j}}=\sum_{m=-M / 2}^{M / 2-1} i m \hat{f}_{m}(r) e^{i 2 \pi m j / M} .
$$

In order to evaluate equation (12), we use a fast Fourier transform algorithm to find $\hat{f}_{m}$, multiply the Fourier coefficient by $i m$, and then take the inverse fast Fourier transform of the series. The computational order of the azimuthal derivative is therefore $\mathcal{O}\left(M \log _{2} M\right)$.

When non-periodic boundary conditions are present, as in the case along the radial direction, the Fourier basis can no longer be used. Popular choices of bases are the Legendre and Chebyshev polynomials. Between these two, the Chebyshev polynomials are usually preferred because of their relation to the cosine function, which makes them easier to compute. Let $T_{n}(r)$ be the $n$-th order Chebyshev polynomial, i.e.,

$$
T_{n}(r)=\cos (n \arccos r) .
$$

Using the Chebyshev-Gauss-Lobatto collocation points,

$$
r_{k}=\cos \left(\frac{\pi k}{N}\right), \quad \text { for } 0 \leq j \leq N
$$

the Chebyshev-Fourier series of any physical quantity $f(r, \phi)$ becomes the double sum

$$
\begin{aligned}
f\left(r_{k}, \phi_{j}\right) & =\sum_{m=-M / 2}^{M / 2-1} \sum_{n=0}^{N} \check{f}_{n, m} T_{n}\left(r_{k}\right) e^{i 2 \pi m j / M} \\
& =\sum_{m=-M / 2}^{M / 2-1} \sum_{n=0}^{N} \check{f}_{n, m} \cos \left(\frac{\pi n k}{N}\right) e^{i 2 \pi m j / M} .
\end{aligned}
$$

We then use a fast discrete cosine transform to obtain the spectral coefficient $\check{f}_{n, m}$ from $\hat{f}_{m}(r)$. When we express the derivative along the radial direction as

$$
\frac{\partial f}{\partial r}=\sum_{m=-M / 2}^{M / 2-1} \sum_{n=0}^{N} \check{f}_{n, m}^{(1)} T_{n}(r) e^{i m \phi},
$$


we can employ the three-term recursive relation between $\check{f}_{n, m}^{(1)}$ and $\check{f}_{n, m}$,

$$
\begin{aligned}
\check{f}_{N, m}^{(1)} & =0 \\
\check{f}_{N-1, m}^{(1)} & =2 N \check{f}_{N, m}, \\
\check{f}_{n, m}^{(1)} & =\frac{\check{f}_{n+2, m}^{(1)}+2(n+1) \check{f}_{n+1, m}}{c_{n}},
\end{aligned}
$$

where $c_{0}=2$ and $c_{n}=1$ otherwise. This recursion relation allows the use of an $\mathcal{O}(N)$ algorithm to calculate the numerical derivatives along the radial direction.

When we use Chebyshev polynomials and Chebyshev-Gauss-Lobatto collocation points, the collocation points are more dense around the boundaries. The grid spacing $\Delta r_{k}=r_{k}-r_{k-1}$ scales with the number of collocation points $N$ as

$$
\Delta r_{k}=\mathcal{O}\left(N^{-2}\right),
$$

when $k$ is close to 0 or $N$. This requires the stability condition for time-stepping (see section 3.5) to be

$$
\Delta t=\mathcal{O}\left(N^{-2}\right),
$$

which is too expensive numerically compared to the one required by the uniform Fourier collocation $\Delta t=\mathcal{O}\left(N^{-1}\right)$. To overcome this restriction, Kosloff, \& Tal-Ezer (1993) proposed the transformation

$$
\tilde{r}_{k} \equiv \frac{\sin ^{-1}\left(\alpha r_{k}\right)}{\sin ^{-1}(\alpha)}
$$

which makes the spacing $\Delta \tilde{r}_{k}=\mathcal{O}\left(N^{-1}\right)$ around the boundaries. The numerical derivatives on the rescaled collocation method can be easily calculated by the chain rule,

$$
\begin{aligned}
\left.\frac{\partial f}{\partial \tilde{r}}\right|_{\tilde{r}_{k}} & =\left.\frac{1}{\left.(d \tilde{r} / d r)\right|_{r_{k}}} \frac{\partial f}{\partial r}\right|_{r_{k}} \\
& =\left.\frac{\sin ^{-1}(\alpha)}{\alpha} \sqrt{1-\left(\alpha \tilde{r}_{k}\right)^{2}} \frac{\partial f}{\partial r}\right|_{r_{k}},
\end{aligned}
$$

where $\left.(\partial f / \partial r)\right|_{r_{k}}$ is given by the usual Chebyshev method (16). Don, \& Solomonoff (1997) showed that the transformation from $r_{k}$ to $\tilde{r}_{k}$ is optimized when

$$
\alpha=\operatorname{sech}\left(\frac{|\ln \epsilon|}{N}\right)
$$

where $\epsilon$ is the machine accuracy. The extra scaling (23) can be done together with the normalization, so the modified Chebyshev collocation takes no extra computational cost. This algorithm is of order $\mathcal{O}\left(N \log _{2} N\right)$ due to the fast cosine transform. Hence, we employ it as the radial collocation method. 


\subsection{Treatment of the Non-Linear Terms}

In low-order numerical methods, one uses the conservative form of hydrodynamic equations to ensure conservation of mass and momentum (LeVeque 1992). This is not the ideal way for pseudospectral methods because the conservative form is sometime numerically unstable (see Peyret 2002, pp. 286-289). To illustrate this, consider two arbitrary functions $u(x)$ and $v(x)$. The derivative of their product has two different but equivalent forms

$$
\begin{aligned}
& C=(u v)^{\prime}, \\
& D=u^{\prime} v+u v^{\prime} .
\end{aligned}
$$

The pseudo-spectral Fourier coefficients (with $M$ collocation points) of $C$ and $D$ are

$$
\begin{aligned}
\hat{C}_{m} & =i m \sum_{p+q=m} \hat{u}_{p} \hat{v}_{q}+i\left[\sum_{p+q=m+M} m \hat{u}_{p} \hat{v}_{q}+\sum_{p+q=m-M} m \hat{u}_{p} \hat{v}_{q}\right], \\
\hat{D}_{m} & =i m \sum_{p+q=m} \hat{u}_{p} \hat{v}_{q}+i\left[\sum_{p+q=m+M}(m+M) \hat{u}_{p} \hat{v}_{q}+\sum_{p+q=m-M}(m-M) \hat{u}_{p} \hat{v}_{q}\right] .
\end{aligned}
$$

The first term in each equation corresponds to the truncated Fourier series. The other terms in the square brackets are therefore due to the aliasing error of pseudo-spectral methods (Boyd 2000, Chapter 11), which may cause numerical instability. One can show that these aliasing terms have opposite signs. The skew symmetric form $\left(\hat{C}_{m}+\hat{D}_{m}\right) / 2$, therefore, has a much smaller aliasing error. Unfortunately, calculating the skew symmetric form increases the number of numerical derivatives and lowers the algorithmic performance by a factor of 2 .

A similar argument is possible for the Chebyshev collocation. However, in that case, the problem becomes more complicated due to the boundary conditions. Botella, \& Peyret (2001) carried out numerical experiments and reported that, without aliasing removal, the convective form is stable for the two different boundary method they tested; the conservative form is unstable for one of their methods.

We carried out some numerical experiments for our algorithm. When the initial conditions are smooth, both conservative and convective forms are stable. However, when an initial perturbation is introduced, the conservative form is always unstable. The spectral coefficients at high wavenumbers in the radial direction grow exponentially even with spectral filtering (see the next subsection). The convective form, on the other hand, is able to reproduce various analytical stability criteria, as shown in section 4 .

Because the convective form gives an approximation that is of the same order as the conservative form, in the present implementations of the numerical algorithm, we use the convective form for both the continuity and the Navier-Stokes equations to ensure numerical stability. 


\section{3. $\quad$ Filtering}

Because of the non-linear character of the Navier-Stokes equations described above, we need to filter out the high-modes in each time step to reduce the aliasing error. Suppose we want to apply a filter in the $\phi$-direction. We first find the Fourier coefficients $\hat{f}_{m}(r)$ according to equation (9). The filtered sum is then

$$
f^{\sigma}\left(r, \phi_{j}\right)=\sum_{m=-M / 2}^{M / 2-1} \sigma_{M / 2}(m) \hat{f}_{m}(r) e^{i 2 \pi m j / M},
$$

where we use the exponential filter

$$
\sigma_{M / 2}(m)=\exp \left(-|\ln \epsilon|\left|\frac{2 m}{M}\right|^{\beta}\right) .
$$

The parameter $\epsilon$ here is again the machine accuracy. We use $\beta \approx M$ so the filtered sum approximates the original function very well and does not reduce the accuracy of the numerical solution. The same filter is used in the radial direction, i.e.,

$$
\sigma_{N}(n)=\exp \left(-|\ln \epsilon|\left(\frac{n}{N}\right)^{\beta}\right)
$$

so the double filtered sum is

$$
f\left(r_{k}, \phi_{j}\right)=\sum_{m=-M / 2}^{M / 2-1} \sum_{n=0}^{N} \sigma_{M / 2}(m) \sigma_{N}(n) \check{f}_{n, m} \cos \left(\frac{\pi n k}{N}\right) e^{i 2 \pi m j / M} .
$$

\subsection{Boundary Conditions}

Solving partial differential equations in a finite domain usually requires Dirichlet or Neumann boundary conditions. When using finite difference methods, one can simply change the values of physical quantities at the boundary points to achieve Dirichlet boundary conditions. Alternatively, one can adjust the "ghost points" to achieve Neumann boundary conditions. Unfortunately, these methods do not generally work for spectral methods. Changing the boundary points (or ghost points) introduces step functions. When discontinuities are present, the spectral coefficients diverge and the spectral methods fail to produce stable solutions.

In our numerical algorithm, we introduce a boundary treatment in the radial direction that is effectively a spatial filter. This method enables each dynamic variable to decay smoothly to its boundary value. For example, if we want to apply a Dirichlet boundary condition at the outer boundary, we choose some smooth monotonic function $g(r)$ such that $g(r) \rightarrow 1$ for interior points of the computational domain and $g(r) \rightarrow 0$ at the boundary. At each time step, we impose the boundary condition by setting

$$
f_{k}^{i} \longrightarrow\left(f_{k}^{i}-f_{0}\right) g\left(\tilde{r}_{k}\right)+f_{0}
$$


where $f_{k}^{i}$ denote the numerical approximation of our function at time $i$ and radial collocation point $k$. This filter brings the function $f$ down to is boundary value $f_{0}$ and produces a numerical boundary layer. Rearranging, the above step is equivalent to setting

$$
f_{k}^{i} \longrightarrow f_{k}^{i}+\left(f_{k}^{i}-f_{0}\right)\left[g\left(r_{k}\right)-1\right] .
$$

Hence the boundary filter is equivalent to adding an extra sink term

$$
\left(f-f_{0}\right) \frac{g(r)-1}{\Delta t}
$$

in the original equation.

We use an exponential filter $g(r)$ that is similar to the spectral filter. To impose an outer boundary condition, we need the monotonically decreasing filter

$$
g(r)=\exp \left[-|\ln \epsilon|\left(\frac{r-r_{\min }}{r_{\max }-r_{\min }}\right)^{\beta}\right] .
$$

On the other hand, for the inner boundary, we use the monotonically increasing filter

$$
g(r)=\exp \left[-|\ln \epsilon|\left(\frac{r_{\max }-r}{r_{\max }-r_{\min }}\right)^{\beta}\right] .
$$

We can change the thickness of the numerical boundary layer by changing the order of the filter $\beta$.

This boundary treatment turns out to be very convenient in modeling non-reflective boundary conditions. When we choose the parameter $\beta$ equal to the number of collocation points, the boundary layer is thick enough to discard any outgoing waves, but thin enough so that the interior solution is not affected significantly.

The addition of ghost a zone is not practical in spectral methods for applying Neumann boundary conditions. This is because Chebyshev polynomials approximate the derivatives based on all the interior points. Godon (1997) presented a method which involved solving for the boundary values of physical quantities, so that their derivatives agree to the boundary conditions. This is effectively a Dirichlet boundary treatment with the boundary values depending on all the interior values at each time step.

To avoid numerical instabilities due to the Neumann boundary conditions, we choose another method that involves applying a spatial filter to each variable after taking the radial derivatives. This is equivalent to replacing $f^{\prime}$ by

$$
f_{k}^{\prime i} \longrightarrow f_{k}^{\prime i}+\left(f_{k}^{\prime i}-f_{0}^{\prime}\right)\left[g\left(r_{k}\right)-1\right] .
$$

This ensures that the first derivatives around the boundaries are smooth. 


\subsection{Time Stepping}

We integrate the hydrodynamics equations with an explicit, three-stage, third-order, RungeKutta method. This method was proposed by Williamson (See Peyret 2002, p.146) and has been widely used in spectral methods. Let

$$
\mathbf{H}=\left(H_{\Sigma}, H_{v_{r}}, H_{v_{\phi}}\right)^{t}
$$

be the right hand sides of hydrodynamic equations (3) - (5). Using superscripts to denote the time discretization as before, the algorithm can be summarized by

$$
\begin{aligned}
\mathbf{V}_{0} & =\mathbf{V}^{n} \\
\mathbf{Q}_{1} & =\Delta t \mathbf{H}\left(\mathbf{V}_{0}\right), \\
\mathbf{V}_{1} & =\mathbf{V}_{0}+\frac{1}{3} \mathbf{Q}_{1}, \\
\mathbf{Q}_{2} & =-\frac{5}{9} \mathbf{Q}_{1}+\Delta t \mathbf{H}\left(\mathbf{V}_{1}\right), \\
\mathbf{V}_{2} & =\mathbf{V}_{1}+\frac{15}{16} \mathbf{Q}_{2}, \\
\mathbf{Q}_{3} & =-\frac{153}{128} \mathbf{Q}_{2}+\Delta t \mathbf{H}\left(\mathbf{V}_{2}\right), \\
\mathbf{V}_{3} & =\mathbf{V}_{2}+\frac{8}{15} \mathbf{Q}_{3}, \\
\mathbf{V}^{n+1} & =\mathbf{V}_{3},
\end{aligned}
$$

where $\Delta t$ is the time step.

Let $\Delta l=\min \left(\Delta \tilde{r}_{k}, \tilde{r}_{k} \Delta \phi_{j}\right)$ be the minimum grid separation. The Courant-Friedrich-Lewy time step $\Delta t_{\mathrm{CFL}}$ and the viscously restricted time step $\Delta t_{\nu_{s}}$ are given by (see also Godon 1997)

$$
\begin{aligned}
\Delta t_{\mathrm{CFL}} & =\min \left(\frac{\Delta l}{c_{s}+|\mathbf{v}|}\right), \\
\Delta t_{\nu_{s}} & =\min \left(\frac{\Delta l^{2}}{\nu}\right) .
\end{aligned}
$$

We choose $\Delta t$ adaptively by requiring

$$
\Delta t \lesssim \min \left(\Delta t_{\mathrm{CFL}}, \Delta t_{\nu}\right) .
$$

\section{Code Validation}

We have validated our numerical algorithm by comparing our numerical results to a number of test problems with analytical solutions. These problems are designed to test the implementation of the different terms in the equations, such as the ones that describe gravity, pressure, and viscosity. In this section, we describe these tests in details. 


\subsection{Free Fall of a Dust Ring}

As a first test, we study the free-fall of an axisymmetric distribution of matter with no angular momentum, pressure, or viscosity. This problem tests both the implementation of gravity and the conservation of mass in the radial direction. We set $v_{\phi}=0, K=0, \nu_{s}=0$, and consider only Newtonian gravity with $\Phi(r)=-G M / r \equiv-1 / r$.

In order to verify our numerical result, we also solve the problem analytically. Using dimensionless quantities so that $G M=1$, we write the conservation of energy for a fluid element initially at $r_{0}$ as

$$
\left(\frac{d r}{d t}\right)^{2}=\frac{2}{r}-\frac{2}{r_{0}}
$$

Integrating over time, we obtain the trajectory $r=r\left(t ; r_{0}\right)$ of each fluid element in the implicit form

$$
\frac{\sqrt{2}}{r_{0} 3 / 2} t=\frac{1}{2} \sin \left(2 \arccos \sqrt{\frac{r}{r_{0}}}\right)+\arccos \sqrt{\frac{r}{r_{0}}} .
$$

We now consider conservation of mass, i.e., at any time we require that

$$
2 \pi r \Sigma(r, t) d r=2 \pi r_{0} \Sigma_{0}\left(r_{0}\right) d r_{0} .
$$

Evaluating $d r_{0}$ by using equation (52), i.e.,

$$
d r_{0}=\frac{r_{0} d r}{\sqrt{r_{0} / r-1}}\left[\frac{3}{2} \sqrt{\frac{2}{r_{0}}} t+\frac{r}{\sqrt{r_{0} / r-1}}\right]^{-1},
$$

the analytical solution for the density becomes

$$
\Sigma(r, t)=\Sigma_{0}\left(r_{0}\right) \frac{r_{0}^{2}}{r}\left[\frac{3 t}{2} \sqrt{\left(\frac{2}{r_{0}}\right)\left(\frac{r_{0}}{r}-1\right)}+r\right]^{-1},
$$

where $r_{0}$ can be obtained implicitly using equation (52).

Although this is an one dimensional problem, we simulate it in the two-dimensional domain $[0.2,1.8] \times[-\pi, \pi]$ with different resolutions. The initial condition is a Gaussian ring

$$
\Sigma_{0}(r, \phi)=\exp \left[-20(r-1)^{2}\right]
$$

with zero initial velocity. At the outer boundary, we use Neumann boundary conditions, i.e., we set $\partial \Sigma /\left.\partial r\right|_{r_{\text {out }}}=0$ and $\partial v_{r} /\left.\partial r\right|_{r_{\text {out }}}=0$ for all times. The boundary filter has order 129 , which gives a boundary layer of approximately 5 collocation points. Because the inner boundary is outflowing and we have neglected viscosity and pressure, we do not need to impose any additional boundary condition there.

Figure 1 compares the simulation to the analytical solution: the open circles denote the numerical results and the solid lines are the analytical solution. The numerical results are indistinguishable 
from the analytical solution. Table 1 lists the maximum error for each resolution at each given time, and demonstrates the ability of our algorithm to converse mass to an accuracy better than a few parts in a million.

\subsection{Propagation of Wavefronts}

In order to test our implementation of the thermal pressure and the numerical spreading of wavefronts, we neglect gravity, set all velocities equal to zero, and simulate the propagation of a sound wave. We use $K=1$ and set the polytropic index $\Gamma$ to $1,4 / 3$, and $5 / 3$. The initial density is

$$
\Sigma_{0}(r, \phi)=1+10^{-3} \exp \left[-60(r-1)^{2}-60 r^{2} \phi^{2}\right]
$$

This is simply a homogeneous hyperbolic problem in polar coordinates. Figure 2 shows six grayscale snapshots of the density for $\Gamma=1$. The resulting sound wave propagates outward as a circle. Note that because of our boundary treatment, the density wave is not reflected back as it propagates close to the boundaries.

To measure the sound speed, we take the density profile at $\phi=0$ and trace the outgoing wavefront. The grid points that correspond to the peak density are plotted as a function of time in Figure 3. Table 2 shows the $\chi^{2}$-fitted sound speed of the simulation. Compared to the analytical sound speed, the error is always within $1 \%$.

\subsection{Rayleigh's Criterion}

We now study the ability of our algorithm to model correctly instabilities in hydrodynamic flows. In the absence of viscosity, the necessary and sufficient condition for a rotating flow with angular velocity $\Omega(r)$ to be stable is

$$
\frac{d}{d r}\left(r^{2} \Omega\right)^{2}>0
$$

which is known as Rayleigh's criterion (Chandrasekhar 1981). Although Rayleigh's criterion usually describes the stability condition in a Couette flow, i.e., for an incompressible fluid between two rotating cylinder, the criterion is also valid for rotating compressible fluids, when the unperturbed radial velocity vanishes everywhere in the flow. This can be achieved by balancing the centrifugal and centripetal acceleration. Let

$$
\kappa^{2}=2 \Omega\left(2 \Omega+r \frac{d \Omega}{d r}\right)
$$

be the square of the epicyclic frequency. Rayleigh's criterion is equivalent to requiring $\kappa^{2}>0$, because $d\left(r^{2} \Omega\right)^{2} / d r=r^{3} \kappa^{2}$.

We define a modified Newtonian gravity with a negative gravitational index $\alpha<0$ such that 
the gravitational acceleration is

$$
g_{r}(r) \equiv-\frac{1}{r^{|\alpha|}} .
$$

The corresponding "Keplerian" velocity $v_{\phi}$ is given by

$$
v_{\phi}=r^{(\alpha+1) / 2} \text {. }
$$

Substituting this into Rayleigh's criterion (eq. [58]), we find that $\alpha=-3$ is the critical stability condition. The growth rate of the instability is

$$
\tau=\operatorname{Im} \sqrt{\kappa^{2}}=\operatorname{Im} \sqrt{(\alpha+3) r^{\alpha-1}} .
$$

Of course, the same result can be obtain by observing that the effective potential

$$
V_{\text {eff }}(r)=\frac{L^{2}}{2 m r^{2}}-\frac{G M m}{(\alpha+1)} r^{\alpha+1}
$$

has no local minimum when $\alpha<-3$, where $L$ is the angular momentum and $m$ is mass of a fluid element.

Four our simulation we use a spatial resolution of $257 \times 64$ collocation points in $(r, \phi)$. The initial conditions are uniform density, $\Sigma_{0}=1$ with the velocity profile (61), and random perturbations in all physical quantities that are of order $10^{-6}$. The boundary conditions enforce $\left.v_{r}\right|_{r_{\min }}=\left.v_{r}\right|_{r_{\max }}=0$.

Because Rayleigh's criterion refers to an one-dimensional problem, we discuss here explicitly the evolution of the $m=0$ mode. This is equivalent to averaging over the azimuthal direction. In the radial direction, we are interested in the perturbations with short wavelengths, i.e., the modes with large wavenumbers. We choose the $k=64$ mode, because it lies at the midpoint of the resolved spectrum and hence does not suffer from significant aliasing errors. Figure 4 shows the evolution of the $k=64, m=0$ mode. When the flow is unstable, the perturbations grow very fast and diverge at around $t=1$. As required by Rayleigh's criterion, flows with $\alpha>-3$ are stable to perturbations.

\subsection{Viscous Spreading of a Fluid Ring}

The spreading of an axisymmetric viscous ring with small kinematic viscosity $\nu_{s}$ (Pringle 1981), has become a standard test for numerical methods including both smoothed particle hydrodynamics (SPH) and grid-based codes. In these simulations, additional structures always appear. Maddison et al. (1996) showed that the concentric rings which appear in SPH simulation using the artificial viscosity given by Monaghan (1992) are numerical artifacts. Later, Speith, \& Kley (2003) shows that spiral structures appear in later times as a result of physical instability, when the full shear viscosity tensor is used.

In the standard solution, it is assumed that $v_{\phi}=r \Omega_{K} \gg v_{r}$ so the viscosity tensor can be approximated by

$$
\sigma_{r r}=\sigma_{\phi \phi}=0
$$




$$
\sigma_{r \phi}=\sigma_{\phi r}=\frac{\partial v_{\phi}}{\partial r}-\frac{v_{\phi}}{r} .
$$

Using conservation of mass, conservation of angular momentum, and the fact that $v_{\phi}$ is always close to the Keplerian value, the analytical solution of density, in our units, becomes

$$
\Sigma(r, t)=\frac{1}{12 \pi \nu_{s} r^{1 / 4} t} \exp \left(-\frac{1+r^{2}}{12 \nu_{s} t}\right) I_{1 / 4}\left(\frac{2 r}{12 \nu_{s} t}\right) .
$$

where $I_{1 / 4}$ is a modified Bessel function.

We perform our simulation with axisymmetric initial conditions. We use a typical value for the viscosity $\nu_{s}=5 \times 10^{-5}$ and set $c_{s}^{2}=0$. The spatial resolution is $257 \times 64$ and the time step is $\Delta t=10^{-3}$. We start at $12 \nu_{s} t=0.01$ (so $t=16.67$ ) and with an initial density given by equation (66) at this time. The boundary conditions are $\left.v_{\phi}\right|_{r_{\max }}=\left.\left(r \Omega_{K}\right)\right|_{r_{\max }}$ and $\left.v_{\phi}\right|_{r_{\min }}=\left.\left(r \Omega_{K}\right)\right|_{r_{\min }}$, which are the ones assumed in the standard solution.

The simulation shows that some concentric rings appear at the beginning because our initial velocity is not the same as those of the standard solution. Figure 5 compares the analytical solution to the numerical solution. The maximum difference between them at $t=216.667$ is 0.0065 .

\section{Conclusion}

We described a numerical method based on a pseudo-spectral algorithm for studying the rapid variability properties of two-dimensional, viscous, hydrodynamic accretion disks. We demonstrated the ability of the spectral method to handle correctly non-reflective boundary conditions and validated the implementation of the algorithm using various test problems.

In all simulations discussed in this paper, we solved only the set of equations consisting of the continuity and Navier-Stokes equations and neglected the energy equation. Instead, we assumed a polytropic equation of state, as adopted in the analytical studies of disk modes (see, e.g., Kato 2001 and references therein), the results of which we will attempt to reproduce numerically . However, it is trivial to extend the algorithm in order to solve simultaneously an energy equation, either for an adiabatic flow, or by approximating the energy losses to radiation as it is often done in accretion-disk models (see, e.g., Shakura \& Sunyaev 1973).

Finally, it is also straightforward to extend the current algorithm to simulate MHD accretion disks. In particular, because of the high order of the spectral method, we can solve directly for the vector potential and not for the magnetic field, in order to guarantee that the resulting field will be free of divergence at each time step. The implementation of the spectral method for MHD flows and the treatment of boundary conditions for the magnetic potential at the outer boundary and at the black-hole horizon will be discussed in the second paper in this series.

C.-K. C. and D.P. acknowledge the support from a NASA ATP grant. F.Ö. acknowledges 
support by NASA through Hubble Fellowship grant HF-01156 from the Space Telescope Science Institute, which is operated by the Association of Universities for Research in Astronomy, Inc., under NASA contract NAS 5-26555.

\section{REFERENCES}

Abramowicz, M.A., \& Kluźniak, W. 2001, A\&A, 374, L19

Armitage, P.J., \& Reynolds, C.S. 2003, MNRAS, 341, 1041

Bonazzola, S., Gourgoulhon, E., \& Marck, J.-A. 1999, J. Comp. Appl. Math. 109, 433

Botella, O., \& Peyret, R. 2001, Int. J. Numer. Methods Fluids 36, 125

Boyd, J.P. 2000, Chebyshev and Fourier Spectral Methods, 2nd Edition (New York: Dover)

Canuto, C., Hussaini, M.Y., Quarteroni, A., \& Zang, T.A. 1987, Spectral Methods in Fluid Dynamics (New York: Springer)

Chandrasekhar, C. 1981, Hydrodynamic and Hydromagnetic Stability (New York: Dover)

Chen, X., \& Taam, R.E. 1994, ApJ, 431, 732

. 1995, ApJ, 441, 354

Don, W.S. 1994, J. of Comp. Phys. 110, 103

Don, W.S., \& Solomonoff, A. 1997, SIAM J. Scien. Comp. 18, no. 4, 1040

Don, W.S., \& Quillen, C.B. 1995, J. of Comp. Phys. 122, 244

Gammie, C.F., \& Balus, S.A. 1994. MNRAS 270, 138

Godon, P. 1997, ApJ 480, 329

Gottlieb, D., \& Orszag, S.A. 1983, Numerical Analyhsis of Spectral Methods: Theory and Applications (Philadelphia: Society for Industrial and Applied Mathematics)

Guo, B.Y. 1998, Spectral Methods and Their Applications (Singapore: World Scientific)

Guo, B.Y., Ma, H.P., Tadmor, E. 2001, SIAM J. Numer. Anal. 39, No. 4, 12254

Hawley, J. F. \& Krolik, J. H. 2001, ApJ, 548, 348

2002, ApJ, 566, 164

Kato, S. 2001, PASJ, 53, 1 
Kosloff, D., \& Tal-Ezer, H. 1993, J. Comp. Phys. 104, 457

LeVeque, R.J. 1992, Numerical Methods for Conservation Laws (Basel: Birkhäuser)

Li, H. 2001, Super Spectral Viscosity Methods for Nonlinear Conservation Laws, Chebysehv Collocation Methods and Their Applications (Thesis)

Maddison, S.T., Murray, J.R., \& Monaghan, J.J. 1996, Publ. Astron. Soc. Aust. 13, 66

McClintock, J.E., \& Remillard R.A. 2004, in Compact Stellar X-ray Sources, eds. W.H.G. Lewin and M. van der Klis (Cambridge: University Press)

Menou, K. 2003, ApJ, 596, 414

Monaghan, J.J. 1992, ARA\&A 30, 534

Mote, P.W., \& O'Neill, A. 2000, Numerical modeling of the global atmosphere in the climate system (Dordrecht; Boston: Kluwer Academic)

Milsom, J.A., \& Taam, R.,E. 1996, MNRAS, 283, 919 .1997, MNRAS, 286, 358

Peyret, R. 2000, Spectral Methods for Incompressible Viscous Flow (New York: Springer)

Pringle, J.E. 1981, ARA\&A 19, 137

Psaltis, D. 2001, Adv. Sp. Res., 24, 481

—. 2004, in X-ray Timing 2003: Rossi and Beyond, eds. P. Kaaret, F.K. Lamb, and J.H. Swank Speith, R., \& Kley, W. 2003, A\&A 399, 395

Strohmayer, T.E. 2001a, ApJ, 554, L169

—. 2001b, ApJ, 552, L49

van der Klis, M. 2000, ARA\&A, 38, 717

Wagoner, R.V. 1999, Physics Reports 311, 259

Wagoner, R.V., Silbergleit, A.S., \& Ortega-Rodriguez, M. 2001, ApJ 559, L25 
Table 1. Maximum errors of the density profile at $\phi=0$ for the free falling Gaussian ring discussed in $\S 4.1$

\begin{tabular}{cccccc}
\hline \hline $\begin{array}{c}\text { Number of } \\
\text { Collocation Points }(r \times \phi)\end{array}$ & $t=0.0$ & $t=0.5$ & $t=1.0$ & $t=1.5$ & $t=2.0$ \\
\hline $33 \times 16$ & 0.0 & $4.395 \times 10^{-6}$ & $8.352 \times 10^{-6}$ & $8.904 \times 10^{-6}$ & $8.729 \times 10^{-6}$ \\
$65 \times 32$ & 0.0 & $2.110 \times 10^{-6}$ & $3.060 \times 10^{-6}$ & $3.411 \times 10^{-6}$ & $3.961 \times 10^{-6}$ \\
$129 \times 64$ & $7.772 \times 10^{-16}$ & $2.038 \times 10^{-6}$ & $3.003 \times 10^{-6}$ & $3.368 \times 10^{-6}$ & $3.913 \times 10^{-6}$ \\
$257 \times 128$ & 0.0 & $2.025 \times 10^{-6}$ & $2.989 \times 10^{-6}$ & $3.352 \times 10^{-6}$ & $3.895 \times 10^{-6}$ \\
\hline
\end{tabular}

Table 2. Analytical and $\chi^{2}$-fitted sound speeds

\begin{tabular}{ccc}
\hline \hline$\Gamma$ & Analytical $c_{s}$ & Numerical $c_{s}$ \\
\hline 1 & 1.0000 & 1.0018 \\
$4 / 3$ & 1.1547 & 1.1599 \\
$5 / 3$ & 1.2910 & 1.2997 \\
\hline
\end{tabular}



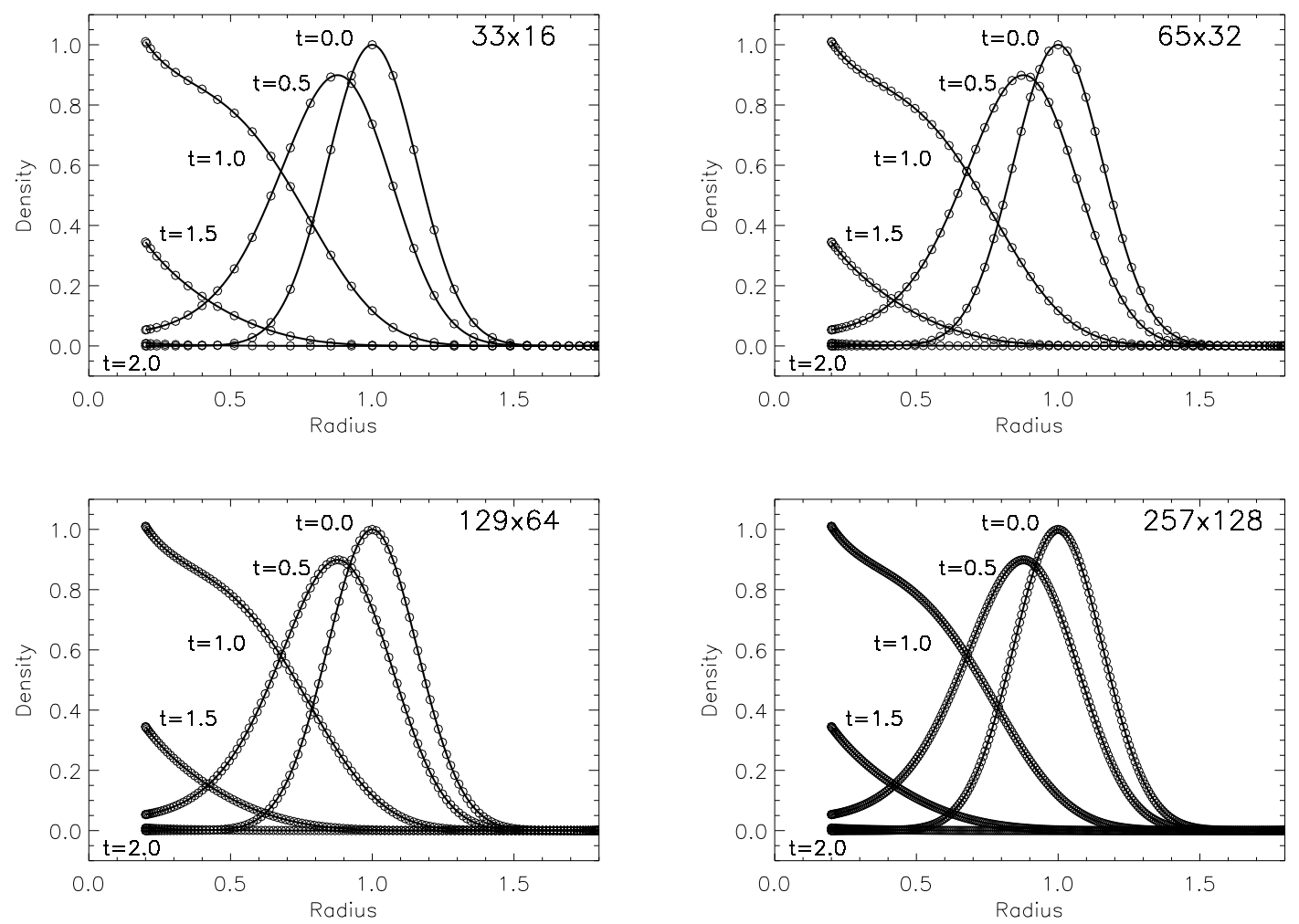

Fig. 1.- The density profile at different times of the free falling Gaussian ring discussed in $\S 4.1$, for different numerical resolutions. The solid lines represent the analytical solution and the open circles represent the numerical results. The errors are of the order of $10^{-6}$. 


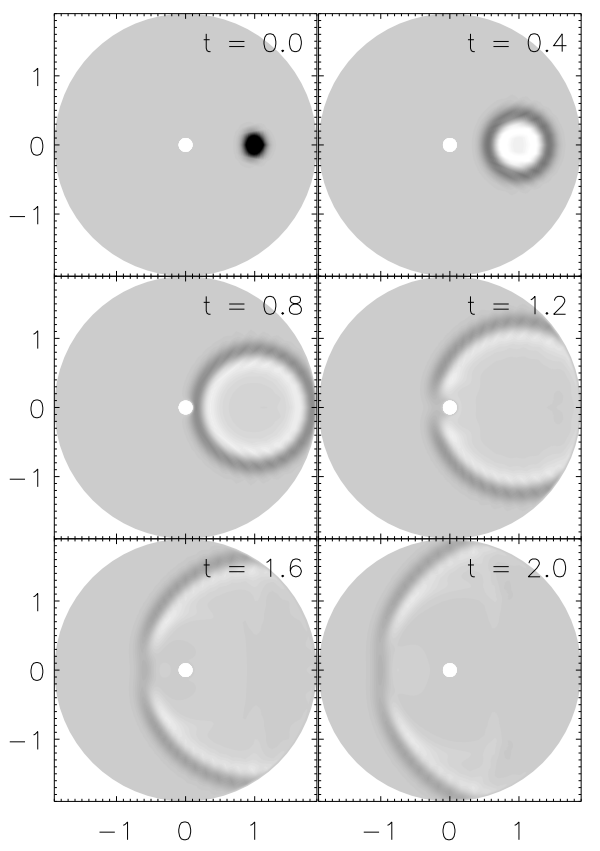

Fig. 2.- The propagation of a sound wave in a uniform static background. The grayscale snapshots correspond to the fluid density.

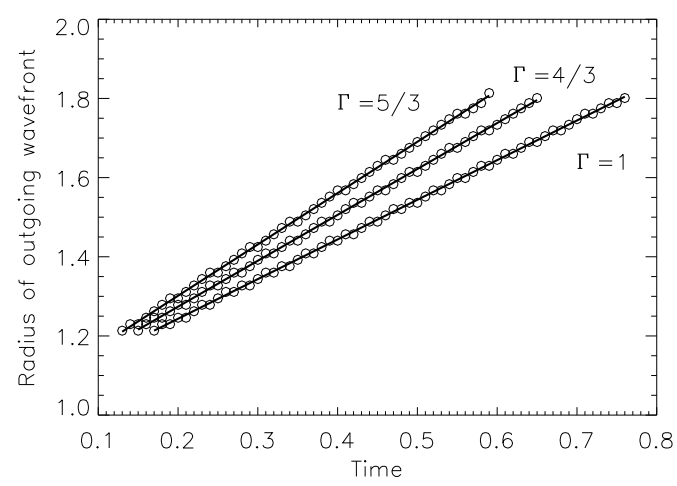

Fig. 3. - The evolution of the radius of a circular sound wave in the absence of gravitational force and viscosity, for three different values of the polytropic index $\Gamma$. The solid lines represent the best-fit linear correlations 


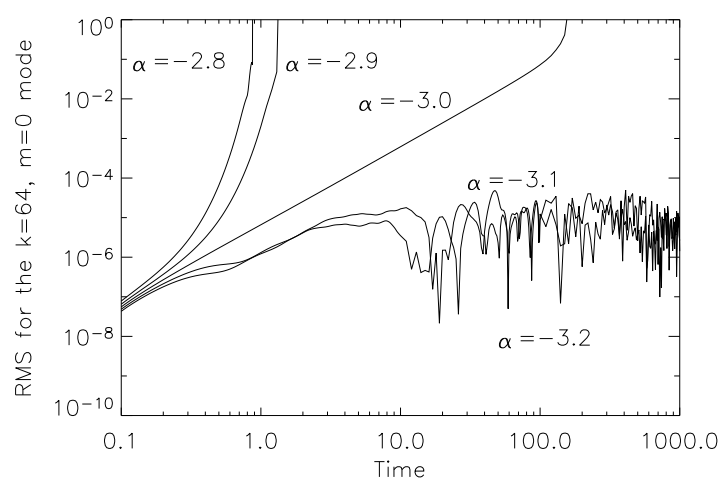

Fig. 4. - The evolution of the $k=64, m=0$ mode for different values of the index $\alpha$ for the study of Rayleigh's criterion discussed in $\S 4.3$.

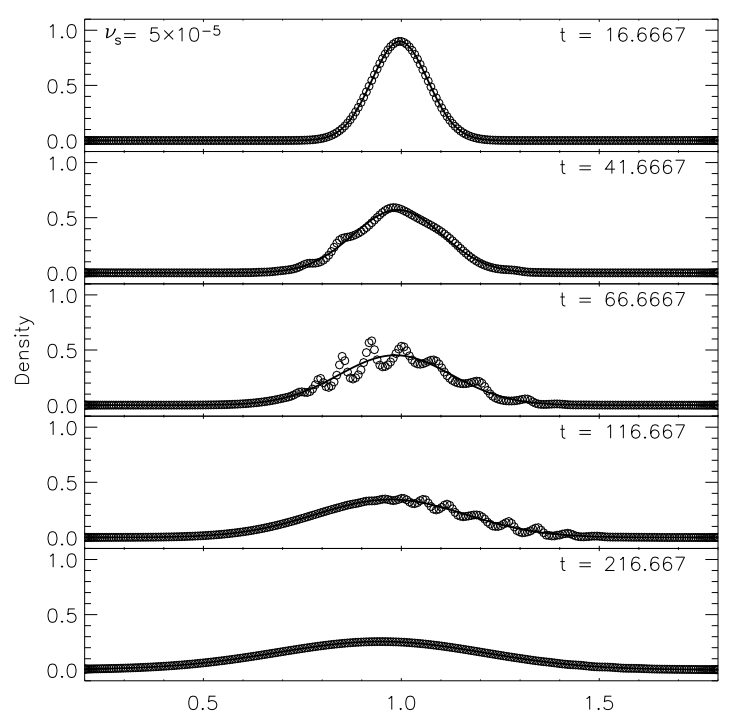

Fig. 5. - The evolution of a viscous spreading ring, for a kinematic viscosity $\nu_{s}=5 \times 10^{-5}$. The solid line is the analytical solution and the circles are numerical solutions. We start the simulation at $t=16.6667$ to avoid the initial $\delta$-function. Note that concentric rings appears at the beginning, but after 32 ring rotations, the concentric rings decay away and we recover the analytic solution. 